\title{
2-烷氧羰基烷基/正丁基-4-芳基-1,5-苯并硫氮杂草类化合物的 合成及抑菌活性的研究
}

\author{
李文红 ${ }^{a, b}$ 刘喜莹 ${ }^{a}$ 张 博 ${ }^{a}$ 李 媛 $*, a$ \\ ( ${ }^{a}$ 河北师范大学化学与材料科学学院 石家庄 050024) \\ ( ${ }^{b}$ 河北工业职业技术学院环境与化学工程系 石家庄 050091)
}

\begin{abstract}
摘要 设计、合成了三类 2 位取代-1,5-苯并硫氮杂草衍生物: 2 -烷氧羰基甲基-4-芳基-1,5-苯并硫氮杂草、2-烷氧羰基乙 基-4-芳基-1,5-苯并硫氮杂䓬和 2-正丙基-4-芳基-1,5-苯并硫氮杂䓬. 所有目标化合物结构经过元素分析, IR, MS, HRMS 及 ${ }^{1} \mathrm{H}$ NMR 确证. 研究了 2-烷氧羰基烷基/正丁基-4-芳基-1,5-苯并硫氮杂草的合成反应条件, 测定了目标化合物的抑真 菌活性. 通过对该类化合物抑真菌活性的评价, 进一步明确乙酯基在 1,5-苯并硫氮杂草 1 的生物活性中所起的作用. 关键词 1,5-苯并硫氮杂草; 合成; 抑真菌活性; 构效关系
\end{abstract}

\section{Synthesis and Antifungal Activity of 2-Carbalkoxyalkyl/(n-butyl)- 4-aryl-1,5-benzothiazepines}

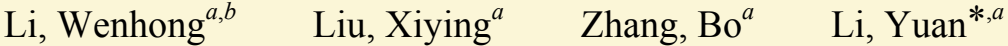 \\ $\left({ }^{a}\right.$ College of Chemistry \& Material Science, Hebei Normal University, Shijiazhuang 050024) \\ $\left({ }^{b}\right.$ Environment and Chemical Engineering, Hebei College of Industry and Technology, Shijiazhuang 050091)
}

\begin{abstract}
A series of novel 2-substituted-1,5-benzothiazepines were synthesized successfully and their structures were determined by elemental analysis, IR, MS/HRMS and ${ }^{1} \mathrm{H}$ NMR. The synthetic conditions of 2-substituted-4-aryl-1,5-benzothiazepines were investigated. The antifungal activities of these 2-substituted-1,5-benzothiazepine derivatives were tested and the results confirmed that the 2-ethyl carboxylate was necessary for antifungal activity in 1,5-benzothiazepine compounds $\mathbf{1}$.
\end{abstract}

Keywords 1,5-benzothiazepine; synthesis; antifungal activities; structure-activity relationship

苯并硫氮杂草是七元杂环的苯并稠环体系, 长期以 来, 关于苯并硫氮杂草类化合物的合成及生理活性的研 究倍受关注. 研究显示, 此类化合物具有多种生理活性, 如: 抗心律失常 ${ }^{[1]}$ 、抑制肿瘤 ${ }^{[2,3]}$ 、抗惊厥作用 ${ }^{[4]}$ 、降血 脂 ${ }^{[5]}$ 以及抑菌活性 ${ }^{[6 \sim 9]}$ 等. 近年来, 本课题组一直开展 1,5-苯并硫氮杂草的合成和抑菌活性的研究 ${ }^{[10,11]}$, 发现 2-甲酸乙酯-4-芳基-1,5-苯并硫氮杂草(1)对新生隐球菌 的抑菌活性很高 ${ }^{[12]}$, 并初步确定了 C(2)位的乙酯基是该 类化合物抗真菌的药效基团之一. 为了深入研究此类杂 草衍生物的构效关系, 进一步明确乙酯基在其抗真菌活 性中的重要地位, 本文围绕杂草 $\mathbf{1}$ 的结构, 从 C(2)位酯 基(乙酯基，甲酯基)的改变、酯基的存在 $(\mathrm{C}(2)$ 上烃基替
换酯基)以及酯基与杂草七元杂环的距离三个方面考虑, 设计、合成了 24 个杂草衍生物, 通过对该类化合物抑真 菌活性的评价, 进一步明确乙酯基在 1,5-苯并硫氮杂草 1 的生物活性中所起的作用.

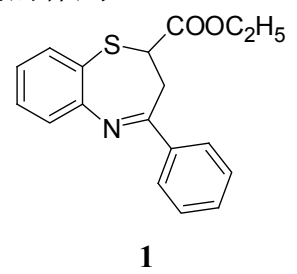

标题化合物的合成路线见 Scheme 1.

*E-mail: liyuanhbsd@163.com

Received October 23, 2013; revised January 18, 2013; published online February 22, 2013.

Project supported by the National Natural Science Foundation of China (No. 20972040), the Research Project of Education Department of Hebei Province of China (No. Q2012001), and the Scientific Research Fund of Hebei College of Industry and Technology (No. Qz1102).

国家自然科学基金(No. 20972040)、河北省教育厅青年基金(No. Q2012001)和河北工业职业技术学院青年基金(No. Qz1102)资助项目. 


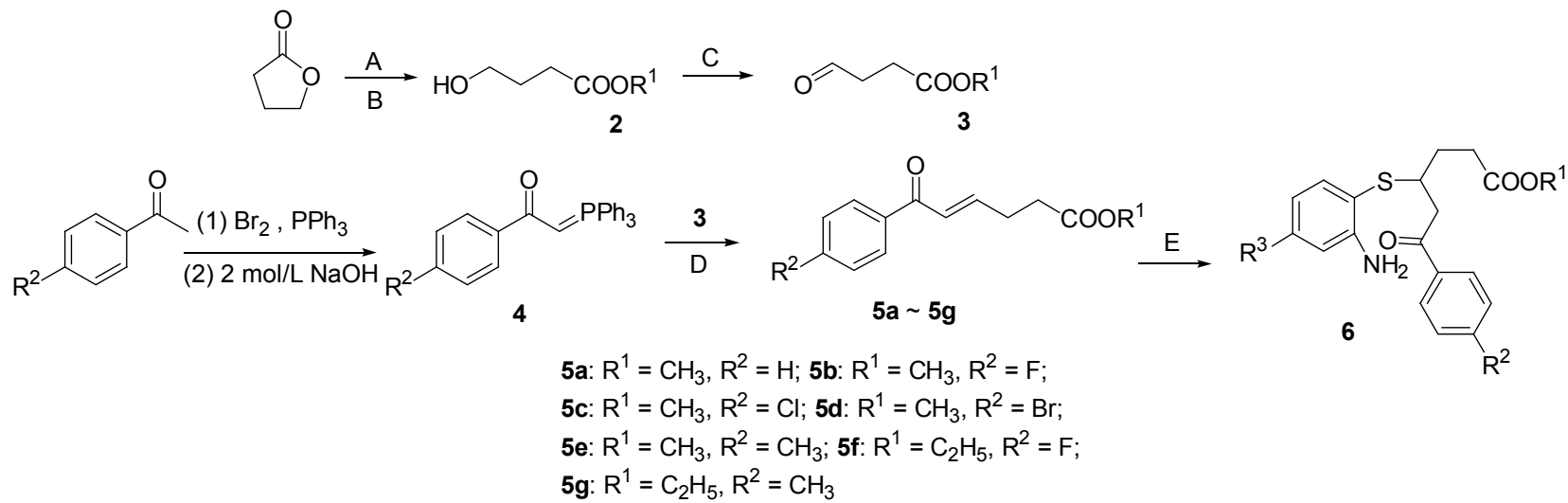

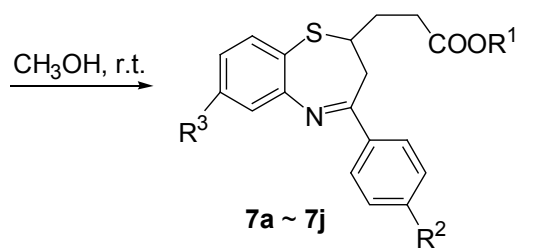

7a: $\mathrm{R}^{1}=\mathrm{CH}_{3}, \mathrm{R}^{2}=\mathrm{H}, \mathrm{R}^{3}=\mathrm{H} ; 7 \mathbf{b}: \mathrm{R}^{1}=\mathrm{CH}_{3}, \mathrm{R}^{2}=\mathrm{F}, \mathrm{R}^{3}=\mathrm{H}$; 7c: $\mathrm{R}^{1}=\mathrm{CH}_{3}, \mathrm{R}^{2}=\mathrm{Cl}, \mathrm{R}^{3}=\mathrm{H} ; \mathbf{7 d}: \mathrm{R}^{1}=\mathrm{CH}_{3}, \mathrm{R}^{2}=\mathrm{Br}, \mathrm{R}^{3}=\mathrm{H}$; 7e: $\mathrm{R}^{1}=\mathrm{CH}_{3}, \mathrm{R}^{2}=\mathrm{CH}_{3}, \mathrm{R}^{3}=\mathrm{H} ; 7 f: \mathrm{R}^{1}=\mathrm{C}_{2} \mathrm{H}_{5}, \mathrm{R}^{2}=\mathrm{F}, \mathrm{R}^{3}=\mathrm{H}$; 7g: $\mathrm{R}^{1}=\mathrm{C}_{2} \mathrm{H}_{5}, \mathrm{R}^{2}=\mathrm{CH}_{3}, \mathrm{R}^{3}=\mathrm{H} ; \mathbf{7 h}: \mathrm{R}^{1}=\mathrm{CH}_{3}, \mathrm{R}^{2}=\mathrm{H}, \mathrm{R}^{3}=\mathrm{Cl}$; 7i: $\mathrm{R}^{1}=\mathrm{CH}_{3}, \mathrm{R}^{2}=\mathrm{CH}_{3}, \mathrm{R}^{3}=\mathrm{Cl} ; 7 \mathrm{j}: \mathrm{R}^{1}=\mathrm{C}_{2} \mathrm{H}_{5}, \mathrm{R}^{2}=\mathrm{H}, \mathrm{R}^{3}=\mathrm{Cl}$

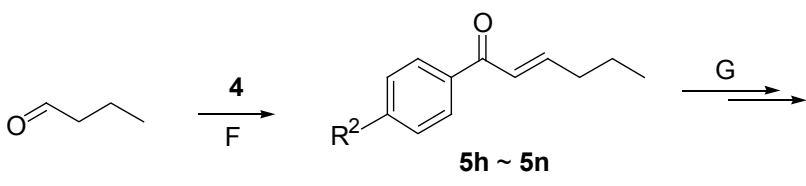

5h: $\mathbf{R}^{2}=\mathrm{H} ; \mathbf{5 i}: \mathrm{R}^{2}=\mathrm{F}$; 5j: $\mathrm{R}^{2}=\mathrm{Cl} ; \mathbf{5 k}: \mathrm{R}^{2}=\mathrm{Br}$; 5I: $\mathrm{R}^{2}=\mathrm{CH}_{3} ; 5 \mathrm{~m}: \mathrm{R}^{2}=\mathrm{OCH}_{3}$; 5n: $\mathrm{R}^{2}=\mathrm{NO}_{2}$

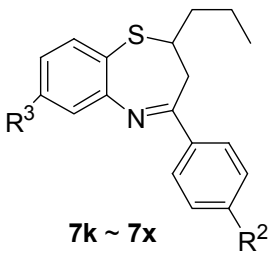

7k: $\mathrm{R}^{2}=\mathrm{H}, \mathrm{R}^{3}=\mathrm{H} ; \mathbf{7 l}: \mathrm{R}^{2}=\mathrm{F}, \mathrm{R}^{3}=\mathrm{H}$;

$7 m: R^{2}=C, R^{3}=H ; 7 n: R^{2}=B r, R^{3}=H$;

7o: $\mathrm{R}^{2}=\mathrm{CH}_{3}, \mathrm{R}^{3}=\mathrm{H} ; 7 \mathrm{p}: \mathrm{R}^{2}=\mathrm{OCH}_{3}, \mathrm{R}^{3}=\mathrm{H}$;

7q: $\mathrm{R}^{2}=\mathrm{NO}_{2}, \mathrm{R}^{3}=\mathrm{H} ; 7 \mathrm{r}: \mathrm{R}^{2}=\mathrm{H}, \mathrm{R}^{3}=\mathrm{Cl}$;

7s: $\mathrm{R}^{2}=\mathrm{F}, \mathrm{R}^{3}=\mathrm{Cl} ; 7 \mathrm{t}: \mathrm{R}^{2}=\mathrm{Cl}, \mathrm{R}^{3}=\mathrm{Cl}$;

7u: $\mathrm{R}^{2}=\mathrm{Br}, \mathrm{R}^{3}=\mathrm{Cl} ; \mathbf{7 v}: \mathrm{R}^{2}=\mathrm{CH}_{3}, \mathrm{R}^{3}=\mathrm{Cl}$;

$7 \mathrm{w}: \mathrm{R}^{2}=\mathrm{OCH}_{3}, \mathrm{R}^{3}=\mathrm{Cl} ; \mathbf{7 x}: \mathrm{R}^{2}=\mathrm{NO}_{2}, \mathrm{R}^{3}=\mathrm{Cl}$

Reagents and conditions: (A) Amberlyst-15, $\mathrm{CH}_{3} \mathrm{OH}$; (B) $\mathrm{C}_{2} \mathrm{H}_{5} \mathrm{OH}$, reflux; (C) $\left(\mathrm{C}_{5} \mathrm{H}_{5} \mathrm{~N}\right)_{2} \cdot \mathrm{CrO}_{3}, \mathrm{CH}_{2} \mathrm{Cl}_{2}, 25{ }^{\circ} \mathrm{C}$; (D) THF, reflux; (E) Aminothiophenol, $\mathrm{CH}_{3} \mathrm{OH}$, reflux/r.t.; (F) THF, $25{ }^{\circ} \mathrm{C}$; (G) Aminothiophenol, $\mathrm{CH}_{3} \mathrm{OH}, 25{ }^{\circ} \mathrm{C}$

\section{Scheme 1}

\section{1 结果与讨论}

\section{1 合成条件研究}

丁内酯在 Amberlyst-15 型酸性离子交换树脂的催化 作用下，与醇发生酯交换反应，生成液态 $\omega$-羊基酸酯 $\mathbf{2}$, 抽滤反应混合物, 用甲醇反复冲洗树脂, 减压蒸馏除去 甲醇, 经气相色谱测试得到中间体 $\mathbf{2}$ 的含量, 杂质未经 过进一步处理, $\omega$-羊基酸酯 2 直接进行下步反应. 在三 氧化铬一吡啶配合物的氧化下, 中间体 2 生成 $\omega$-甲酰基 酸酯 3. 该氧化反应注意以下反应条件: 需要氮气保护, 反应仪器和原料都要保证无水, 滴加 $\omega$-羊基酸酯 $\mathbf{2}$ 的二 氯甲烷溶液, 室温反应. 如果该反应不能兼顾以上条件, 则直接导致反应产率下降，甚至失败.

苯乙酮经过溴代, 再与磷盐反应, 在碱性条件下脱 溴得到 Wittig 试剂 4. 中间体 4 与中间体 3 或丁醛反
应, 四氢呋喃作溶剂, 加热回流 $16 \mathrm{~h}$, 得到 $\alpha, \beta$-不饱和 酮中间体 5. 研究表明, 苯环上有供电子基的 Wittig 试 剂 4 更容易发生上述反应，其原因是苯环上的供电子基 通过共轭效应增强了 Wittig 试剂的亲核性, 从而促进了 缩合反应的进行. 此外, Wittig 试剂与丁醛反应比较容 易进行，而与其结构类似物一分子末端具有酯基的 $\omega$ 羊基酸酯 3 反应却比较困难，可能是因为在制备 Wittig 试剂的过程中对产物都没有进行提纯, 杂质影响了 Wittig 试剂与 $\omega$-差基酸酯的加成反应.

由中间体 5 与邻氨基硫酚反应生成中间体 $\mathbf{6}$, 再脱 水生成目标化合物 7. 合成中间体 6 的反应速度较快, 一般反应 $10 \mathrm{~min}$ 左右即可完成. 中间体 $\mathbf{6}$ 脱水转化成 杂草 7 时反应速度较慢, 并且需要酸催化. 本文研究了 醋酸、对甲基苯磺酸、四氯化钛、蒙脱土以及酸性离子 交换树脂对该反应的催化作用，发现加入催化量 $(5 \%$ 
$\mathrm{moL}$ )的对甲苯磺酸时反应产率最高; 研究还发现, 用甲 醇作溶剂更有利于杂草 7 的合成, 加热使该反应速度加 快, 但是加热时间过长则副产物增多, 一般以加热回流 $4 \mathrm{~h}$ 为宜.

\section{2 光谱特征}

从表征数据中可以得出, 合成的 24 个 2 -烷氧羊基 烷基/正丁基-4-芳基-1,5-苯并硫氮杂草的红外图谱中, $\mathrm{C}=\mathrm{N}$ 双键的位置在 $1598 \sim 1616 \mathrm{~cm}^{-1}, \mathrm{C}=\mathrm{O}$ 双键的位 置在 $1728 \sim 1736 \mathrm{~cm}^{-1}, \mathrm{C}=\mathrm{O}$ 双键的吸收比较强. ${ }^{1} \mathrm{H}$ NMR 谱图中芳环的化学氢位移 $(\delta)$ 值在 6.90 8.00 之间; 杂草七元环上的三个氢构成 $\mathrm{ABX}$ 体系氢谱, 目标化合 物 7a $\sim 7 \mathbf{j}$ 的 ${ }^{1} \mathrm{H}$ NMR 谱图中没有出现典型的 $\mathrm{ABX}$ 体系 的特征峰 (4, 4, 4 重峰), 原因是七元杂环 $-\mathrm{CH}_{2}$ 的质子和 支链上的质子峰重合而得到多重峰. 目标化合物 $7 \mathbf{k} \sim$ $7 \mathbf{x}$ 的 ${ }^{1} \mathrm{H}$ NMR 谱图中出现 ABX 体系的特征峰, 其中两 个氢裂分成 $(4,4)$ 重峰, 另一个氢受七元杂环 2 位碳上支 链烷基氢的影响, 裂分成多重峰. 质谱和高分辨质谱出 现的是 $\mathrm{M}^{+}+1$ 峰.

\section{3 抑菌活性测试}

以合成的 24 个目标化合物 $7 \mathbf{a} \sim 7 \mathbf{x}$ 作为供试样品, 分别用 DMSO 将其配成溶液, 用微量加样器加到滤纸 片上, 使每片含量分别为 $200 \mu \mathrm{g}$. 用滤纸片法测定其对 C. albicans (ATCC10231), C. neoformans (ATCC34874) 和 C. neoformans (Clinical strains) 的抑真菌活性. 抑菌测 试结果为目标化合物 $7 \mathbf{a} \sim 7 \mathbf{x}$ 的抑菌圈为 6 , 表明该类化 合物均没有抑菌活性.

对照样品 2-甲酸乙酯-4-芳基-1,5-苯并硫氮杂草系 列化合物中抑菌活性最好样品的抑菌圈达到 $20 \sim 30$ $\mathrm{mm}$, 而本文的 24 个目标化合物, 对白色念株菌、新生 隐球菌(标准株和临床株)几乎没有抑菌活性. 这一结果 说明杂草 1 的 2 位上的乙酯基对其抗真菌活性是必要的 基团，去掉该基团或者用甲酯基代替乙酯基以及拉大乙 酯基与七元杂环的距离都会大大降低杂草 $\mathbf{1}$ 对真菌的抗 菌活性. 根据药物化学的基本原理, 药物的生物活性与 其空间构型有很大的关系, 取消活性基团-乙酯基或改 变了该活性基团的位置, 都会导致此类杂草化合物的抑 菌活性大大下降甚至丧失. 抑菌活性测试结果进一步验 证了乙酯基在杂苫 1 中的药效基团地位.

\section{2 结论}

为了考察杂草 1 分子中乙酯基对其抗真菌活性的影 响, 从杂草 1 的结构出发, 设计合成了 24 个目标化合物: 2-烷氧羰基甲基-4-芳基-1,5-苯并硫氮杂草、2-烷氧羰基 乙基-4-芳基-1,5-苯并硫氮杂草和 2-正丁基-4-芳基-1,5-
苯并硫氮杂草, 考察了它们对白色念珠菌、新生隐球菌 的抑菌活性. 研究表明, 当杂草 $\mathbf{1}$ 的 C(2)位的乙酯基被 甲酯基、烷基取代或乙酯基不直接与 $\mathrm{C}(2)$ 相连时, 其抗 真菌活性大大降低, 该结论进一步说明了乙酯基是杂草 1 的药效基团, 并且确定了乙酯基在该活性分子中的确 切位置. 以上研究结果为筛选高活性化合物提供了有价 值的参考.

\section{3 实验部分}

\section{1 仪器与试剂}

X-4 型数字显示显微熔点测定仪(温度计未校正); varioELIII 型元素分析仪; Vertex 70 FT-IR 型红外分光光 度计; AVIII500Q Bruker 超导核磁共振仪; xevo 质谱仪; Apex Ultra 傅里叶变换质谱仪. 所用试剂为化学纯或分 析纯, 全部加干燥剂处理.

\section{2 实验方法}

\subsubsection{4-羟基-丁酸甲酯 (2a) 的合成}

在 $100 \mathrm{~mL}$ 锥形瓶中, 依次加入 $\gamma$-丁内酯 $0.86 \mathrm{~g}$ (0.01 mol), 树脂 $25 \mathrm{~g} 、 25 \mathrm{~mL}$ 甲醇, 密封. 每隔 $4 \mathrm{~h}$ 振荡 $2 \mathrm{~min}, 12 \mathrm{~h}$ 后反应结束, 抽滤, 用 $25 \mathrm{~mL}$ 甲醇洗涤树脂 三次, 浓缩滤液得到 2a, 气相色谱测试, 2a 含量 70\% $79 \%{ }^{[13]}$.

\subsubsection{4-羟基一丁酸乙酯 $(\mathbf{2 b})$ 的合成}

在 $250 \mathrm{~mL}$ 圆底烧瓶中, 依次加入 $\gamma$-丁内酯 $8.60 \mathrm{~g}$ $(0.1 \mathrm{~mol}) 、 150 \mathrm{~mL}$ 无水乙醇, 然后滴加 $9.2 \mathrm{~g}$ 浓硫酸, 加 热回流 $2 \mathrm{~h}$, 浓缩, 加入碳酸钠调 $\mathrm{pH}$ 值为 $7 \sim 8$, 然后用 无水乙醚萃取三次, 无水硫酸镁干燥. 旋蒸得到得无色 油状液体 2b. 气相色谱测定, $2 \mathrm{~b}$ 含量 $60 \%{ }^{[14]}$.

\subsubsection{4-氧代-丁酸酯醛 (3)的合成}

以 3a 为例, 在 $500 \mathrm{~mL}$ 四口瓶中, 氮气保护下加入 $150 \mathrm{~mL}$ 二氯甲烷、三氧化铬 $6.0 \mathrm{~g}(0.06 \mathrm{mmol})$, 搅拌下 加入吡啶 $9.60 \mathrm{~mL}(0.12 \mathrm{mmol}), 20 \mathrm{~min}$ 后, 逐滴加入 2a $1.58 \mathrm{~g}(0.01 \mathrm{mmol})$ 的二氯甲烷混合液, 反应液由红褐色 变成深棕黑色, 继续搅拌 $1 \mathrm{~h}$, 停止反应. 得到粘稠混合 物, 用 $200 \mathrm{~mL}$ 无水乙醚萃取反应液, 依次用 $5 \%$ 氢氧 化钠溶液、 $5 \%$ 盐酸溶液、 $5 \%$ 碳酸钠溶液、饱和氯化 钠溶液洗涤, 无水硫酸镁干燥. 抽滤, 浓缩, 得淡黄色 油状液体, 经气相测定, $3 \mathrm{a}$ 含量 $50 \%$.

\subsubsection{2-氧-2-苯乙基-三苯基磷烷(4)的合成}

中间体 4 的合成参见文献[15].

\subsubsection{6-芳基-6-氧代-4-已烯酸酯/已烯(5)的合成}

以 $5 \mathrm{a}$ 为例, 在 $100 \mathrm{~mL}$ 圆底烧瓶中, 加入 $4 \mathrm{a} 1.90 \mathrm{~g}$ (0.005 mol), 3a 混合液 $1.16 \mathrm{~g}$ (含纯 3a $0.005 \mathrm{~mol}$ ), $50 \mathrm{~mL}$ 四氢呋喃, 搅拌均匀后, 加热回流 $16 \mathrm{~h}$. 经 TLC 监测, 
反应结束, 浓缩反应液, 得到产物 $\mathbf{5 a}$. 依照此法合成 $5 \mathrm{~b} \sim 5 \mathrm{~g}$.

以 $\mathbf{5 h}$ 为例, 在 $100 \mathrm{~mL}$ 圆底烧瓶中, 加入 $4 \mathbf{a} 1.90 \mathrm{~g}$ (0.005 mol), $0.36 \mathrm{~g}$ 正丁醛 $(0.005 \mathrm{~mol}), 50 \mathrm{~mL}$ 四氢呋喃, 室温搅拌反应 $16 \mathrm{~h}$. 经 TLC 监测, 反应结束, 浓缩反应 液, 得产物 $\mathbf{5 h}$. 依照此法合成 $\mathbf{5 i} \sim \mathbf{5 n}$.

3.2.6 2-烷氧羰基烷基/正丁酯-4-芳基-1.5-苯并硫氮 杂草(7)的合成

以 7a 为例, 在 $100 \mathrm{~mL}$ 圆底烧瓶中, 加入 $5 \mathrm{a} 1.10 \mathrm{~g}$ (0.005 mol), 邻氨基苯硫酚 $0.62 \mathrm{~g}(0.005 \mathrm{~mol}), 25 \mathrm{~mL}$ $\mathrm{CH}_{3} \mathrm{OH}$, 室温下搅拌, 经 TCL 监测, 10 min 后 $\mathbf{5 a}$ 大部分 转化成了 $6 \mathrm{a}$, 继续反应 $4 \mathrm{~h}, \mathbf{6 a}$ 几乎全部转化成了杂草 7a. 经硅胶柱柱层析分离, 洗脱剂为: $V$ (石油醚) : $V($ 乙 酸乙酯) $=15: 1$, 得到 $1.3 \mathrm{~g} 7 \mathbf{7 a}$. 依照此法得到 $7 \mathbf{b} \sim 7 \mathbf{x}$.

7a: 白色固体, 产率 90\%. m.p. $42{ }^{\circ} \mathrm{C} ;{ }^{1} \mathrm{H}$ NMR $\left(\mathrm{CDCl}_{3}, 500 \mathrm{MHz}\right) \delta: 7.07 \sim 8.04(\mathrm{~m}, 9 \mathrm{H}), 3.84 \sim 3.90(\mathrm{~m}$, $1 \mathrm{H}), 3.69$ (s, 3H), 3.15 (q, $J=5.5 \mathrm{~Hz}, 1 \mathrm{H}), 2.46 \sim 2.61(\mathrm{~m}$, $3 \mathrm{H}), 1.88 \sim 1.96(\mathrm{~m}, 1 \mathrm{H}), 1.98 \sim 2.05(\mathrm{~m}, 1 \mathrm{H})$; IR $(\mathrm{KBr}) v$ : 1609.9, $1734.0 \mathrm{~cm}^{-1}$; MS (30 eV) $\mathrm{m} / z(\%): 326.04(\mathrm{M}+$ $\left.\mathrm{H}^{+}, 100\right), 327.07$ (21). Anal. calcd for $\mathrm{C}_{19} \mathrm{H}_{19} \mathrm{NO}_{2} \mathrm{~S}$ : C 76.82, H 6.81, N 4.98; found C 76.80, H 6.92, N 4.89.

7b: 白色固体, 产率 95\%. m.p. 84 85 ${ }^{\circ} \mathrm{C} ;{ }^{1} \mathrm{H}$ NMR $\left(\mathrm{CDCl}_{3}, 500 \mathrm{MHz}\right) \delta: 7.11 \sim 8.11(\mathrm{~m}, 8 \mathrm{H}), 3.83 \sim 3.90(\mathrm{~m}$, $1 \mathrm{H}), 3.70(\mathrm{~s}, 3 \mathrm{H}), 3.15$ (q, $J=5.5 \mathrm{~Hz}, 1 \mathrm{H}), 2.47 \sim 2.61(\mathrm{~m}$, $3 \mathrm{H}), 1.87 \sim 1.93(\mathrm{~m}, 1 \mathrm{H}) 1.95 \sim 2.05(\mathrm{~m}, 1 \mathrm{H})$; IR $(\mathrm{KBr}) v$ : $1600.8,1735.8 \mathrm{~cm}^{-1}$; MS (30 eV) $\mathrm{m} / z(\%): 344.10(\mathrm{M}+$ $\left.\mathrm{H}^{+}, 100\right)$. Anal. calcd for $\mathrm{C}_{19} \mathrm{H}_{18} \mathrm{FNO}_{2} \mathrm{~S}$ : C 66.45, H 5.28, N 5.53; found C 66.40, H 5.19, N 5.68.

7c: 白色固体, 产率 90\%. m.p. 93 95 ${ }^{\circ} \mathrm{C} ;{ }^{1} \mathrm{H}$ NMR $\left(\mathrm{CDCl}_{3}, 500 \mathrm{MHz}\right) \delta: 7.08 \sim 7.99(\mathrm{~m}, 8 \mathrm{H}), 3.82 \sim 3.88(\mathrm{~m}$, $1 \mathrm{H}), 3.70$ (s, 3H), 3.09 (q, $J=5.5 \mathrm{~Hz}, 1 \mathrm{H}), 2.44 \sim 2.58(\mathrm{~m}$, $3 \mathrm{H}), 1.86 \sim 1.93(\mathrm{~m}, 1 \mathrm{H}), 1.94 \sim 2.05(\mathrm{~m}, 1 \mathrm{H})$; IR $(\mathrm{KBr}) v$ : 1609.2, $1736.3 \mathrm{~cm}^{-1}$; MS (30 eV) $\mathrm{m} / z(\%): 359.77(\mathrm{M}+$ $\mathrm{H}^{+}, 100$ ), 361.77 (33). Anal. calcd for $\mathrm{C}_{19} \mathrm{H}_{18} \mathrm{NO}_{2} \mathrm{SCl}$ : C 63.41, H 5.04, N 3.89; found C 63.40, H 5.12, N 3.98 .

7d: 白色固体, 产率 94\%. m.p. $97 \sim 98{ }^{\circ} \mathrm{C} ;{ }^{1} \mathrm{H}$ NMR $\left(\mathrm{CDCl}_{3}, 500 \mathrm{MHz}\right) \delta$ : $7.08 \sim 7.92(\mathrm{~m}, 8 \mathrm{H}), 3.81 \sim$ $3.88(\mathrm{~m}, 1 \mathrm{H}), 3.70(\mathrm{~s}, 3 \mathrm{H}), 3.09$ (q, $J=5.0 \mathrm{~Hz}, 1 \mathrm{H}), 2.44 \sim$ $2.60(\mathrm{~m}, 3 \mathrm{H}), 1.87 \sim 1.94(\mathrm{~m}, 1 \mathrm{H}), 1.98 \sim 2.05(\mathrm{~m}, 1 \mathrm{H})$; IR (KBr) v: 1602.7, $1735.8 \mathrm{~cm}^{-1}$; HRMS calcd for $\mathrm{C}_{19} \mathrm{H}_{19} \mathrm{BrNO}_{2} \mathrm{~S}$ 404.0314, found 404.0314.

7e: 白色固体, 产率 $96 \%$. m.p. $78 \sim 80{ }^{\circ} \mathrm{C} ;{ }^{1} \mathrm{H}$ NMR $\left(\mathrm{CDCl}_{3}, 500 \mathrm{MHz}\right) \delta$ : $7.05 \sim 7.94(\mathrm{~m}, 8 \mathrm{H}), 3.81 \sim$ $3.87(\mathrm{~m}, 1 \mathrm{H}), 3.69(\mathrm{~s}, 3 \mathrm{H}), 3.13(\mathrm{q}, J=5.0 \mathrm{~Hz}, 1 \mathrm{H}), 2.43 \sim$ $2.60(\mathrm{~m}, 3 \mathrm{H}), 2.42(\mathrm{~s}, 3 \mathrm{H}), 1.87 \sim 1.94(\mathrm{~m}, 1 \mathrm{H}), 1.97 \sim$ $2.04(\mathrm{~m}, 1 \mathrm{H})$; IR (KBr) v: 1616.2, $1733.9 \mathrm{~cm}^{-1}$; MS (30 eV) $m / z(\%): 339.81\left(\mathrm{M}+\mathrm{H}^{+}, 100\right), 340.79(80), 341.79$ (64). Anal. calcd for $\mathrm{C}_{20} \mathrm{H}_{21} \mathrm{NO}_{2} \mathrm{~S}$ : C 70.77, $\mathrm{H} 6.24, \mathrm{~N}$ 4.13; found C 70.75, H 6.16, N 4.24.

7f: 白色固体, 产率 93\%. m.p. 51 52 ${ }^{\circ} \mathrm{C} ;{ }^{1} \mathrm{H}$ NMR $\left(\mathrm{CDCl}_{3}, 500 \mathrm{MHz}\right) \delta: 7.07 \sim 8.06(\mathrm{~m}, 8 \mathrm{H}), 4.12 \sim 4.17(\mathrm{~m}$, $2 \mathrm{H}), 3.83 \sim 3.88(\mathrm{~m}, 1 \mathrm{H}), 3.11(\mathrm{q}, J=5.5 \mathrm{~Hz}, 1 \mathrm{H}), 2.45 \sim$ $2.59(\mathrm{~m}, 3 \mathrm{H}), 1.87 \sim 1.95(\mathrm{~m}, 1 \mathrm{H}), 1.97 \sim 2.04(\mathrm{~m}, 1 \mathrm{H})$, $1.27(\mathrm{t}, J=7.5 \mathrm{~Hz}, 3 \mathrm{H})$; IR (KBr) $v: 1612.4,1728.1 \mathrm{~cm}^{-1}$; MS (30 eV) $m / z(\%): 358.13\left(\mathrm{M}+\mathrm{H}^{+}, 100\right)$. Anal. calcd for $\mathrm{C}_{20} \mathrm{H}_{20} \mathrm{FNO}_{2} \mathrm{~S}$ : C 67.20, H 5.64, N 5.32; found C 67.32, H 5.62.16, N 5.38 .

7g: 白色固体, 产率 96\%. m.p. $66 \sim 67{ }^{\circ} \mathrm{C} ;{ }^{1} \mathrm{H}$ NMR $\left(\mathrm{CDCl}_{3}, 500 \mathrm{MHz}\right) \delta: 7.05 \sim 7.94(\mathrm{~m}, 8 \mathrm{H}), 4.15$ (q, $J=7.0$ $\mathrm{Hz}, 2 \mathrm{H}), 3.82 \sim 3.87(\mathrm{~m}, 1 \mathrm{H}), 3.12(\mathrm{q}, J=5.0 \mathrm{~Hz}, 1 \mathrm{H})$, $2.46 \sim 2.58(\mathrm{~m}, 3 \mathrm{H}), 2.42(\mathrm{~s}, 3 \mathrm{H}), 1.87 \sim 1.95(\mathrm{~m}, 1 \mathrm{H})$, $1.97 \sim 2.04(\mathrm{~m}, 1 \mathrm{H}), 1.26(\mathrm{t}, J=7.0 \mathrm{~Hz}, 3 \mathrm{H})$; IR (KBr) $v$ : 1598.9, $1728.1 \mathrm{~cm}^{-1}$; MS (30 eV) $\mathrm{m} / z(\%): 354.2\left(\mathrm{M}+\mathrm{H}^{+}\right.$, 100). Anal. calcd for $\mathrm{C}_{21} \mathrm{H}_{23} \mathrm{NO}_{2} \mathrm{~S}: \mathrm{C} 71.36, \mathrm{H} 6.56, \mathrm{~N}$ 3.96; found C 71.38, H 6.62, N 3.99.

7h: 白色固体, 产率 $90 \%$. m.p. $100 \sim 102{ }^{\circ} \mathrm{C} ;{ }^{1} \mathrm{H}$ NMR $\left(\mathrm{CDCl}_{3}, 500 \mathrm{MHz}\right) \delta: 7.10 \sim 8.09(\mathrm{~m}, 8 \mathrm{H}), 3.86 \sim$ $3.91(\mathrm{~m}, 1 \mathrm{H}), 3.70(\mathrm{~s}, 3 \mathrm{H}), 3.23(\mathrm{q}, J=2.5 \mathrm{~Hz}, 1 \mathrm{H}), 2.46 \sim$ $2.58(\mathrm{~m}, 3 \mathrm{H}), 1.86 \sim 1.93(\mathrm{~m}, 1 \mathrm{H}), 1.99 \sim 2.06(\mathrm{~m}, 1 \mathrm{H})$; IR (KBr) $v: 1608.5,1733.9 \mathrm{~cm}^{-1}$; MS (30 eV) $\mathrm{m} / z(\%)$ : $360.12\left(\mathrm{M}+\mathrm{H}^{+}, 100\right), 362.11$ (38). Anal. calcd for $\mathrm{C}_{19} \mathrm{H}_{18} \mathrm{NO}_{2} \mathrm{SCl}$ : C 63.41, H 5.04, N 3.89; found C 63.46, H 5.15, N 3.97.

7i: 白色固体, 产率 95\%. m.p. $123 \sim 124{ }^{\circ} \mathrm{C} ;{ }^{1} \mathrm{H}$ NMR $\left(\mathrm{CDCl}_{3}, 500 \mathrm{MHz}\right) \delta: 7.03 \sim 7.92(\mathrm{~m}, 7 \mathrm{H}), 3.81 \sim$ $3.87(\mathrm{~m}, 1 \mathrm{H}), 3.69(\mathrm{~s}, 3 \mathrm{H}), 3.14(\mathrm{q}, J=5.0 \mathrm{~Hz}, 1 \mathrm{H}), 2.41 \sim$ $2.56(\mathrm{~m}, 3 \mathrm{H}), 2.43(\mathrm{~s}, 3 \mathrm{H}), 1.85 \sim 1.93(\mathrm{~m}, 1 \mathrm{H}), 1.97 \sim$ 2.04 (m, 1H); IR (KBr) v: 1602.7, $1732.0 \mathrm{~cm}^{-1}$; MS (30 eV) $m / z(\%): 374.0\left(\mathrm{M}+\mathrm{H}^{+}, 100\right)$. Anal. calcd for $\mathrm{C}_{20} \mathrm{H}_{20} \mathrm{NO}_{2} \mathrm{SCl}$ : C 64.25, H 5.39, N 3.75; found C 64.29, H 5.42, N 3.79 .

7j: 白色固体，产率 94\%. m.p. $40 \sim 42{ }^{\circ} \mathrm{C} ;{ }^{1} \mathrm{H}$ NMR $\left(\mathrm{CDCl}_{3}, 500 \mathrm{MHz}\right) \delta: 7.06 \sim 8.04(\mathrm{~m}, 8 \mathrm{H}), 4.15$ (q, $J=7.0 \mathrm{~Hz}, 2 \mathrm{H}), 3.85 \sim 3.91(\mathrm{~m}, 1 \mathrm{H}), 3.18(\mathrm{q}, J=5.0 \mathrm{~Hz}$, $1 \mathrm{H}), 2.44 \sim 2.56(\mathrm{~m}, 3 \mathrm{H}), 1.86 \sim 1.94(\mathrm{~m}, 1 \mathrm{H}), 1.98 \sim 2.04$ (m, 1H), 1.27 (t, $J=7.5 \mathrm{~Hz}, 3 \mathrm{H})$; IR (KBr) v: 1606.6, $1729.2 \mathrm{~cm}^{-1}$; MS (30 eV) $\mathrm{m} / \mathrm{z}(\%): 374.1\left(\mathrm{M}+\mathrm{H}^{+}, 100\right)$. Anal. calcd for $\mathrm{C}_{20} \mathrm{H}_{20} \mathrm{NO}_{2} \mathrm{SCl}: \mathrm{C} 64.25, \mathrm{H} 5.39, \mathrm{~N} 3.75$; found C 64.29, H 5.42, N 3.79.

7k: 白色固体, 产率 $85 \%$. m.p. $39 \sim 40{ }^{\circ} \mathrm{C} ;{ }^{1} \mathrm{H}$ NMR 
$\left(\mathrm{CDCl}_{3}, 500 \mathrm{MHz}\right) \delta: 7.06 \sim 8.04(\mathrm{~m}, 9 \mathrm{H}), 3.84 \sim 3.90(\mathrm{~m}$, $1 \mathrm{H}), 3.06(\mathrm{dd}, J=5.0,13.0 \mathrm{~Hz}, 1 \mathrm{H}), 2.52(\mathrm{dd}, J=12.0$, $13.0 \mathrm{~Hz}, 1 \mathrm{H}), 1.38 \sim 1.46(\mathrm{~m}, 2 \mathrm{H}), 1.51 \sim 1.58(\mathrm{~m}, 1 \mathrm{H})$, $1.38 \sim 1.46(\mathrm{~m}, 1 \mathrm{H}), 0.93$ (t, $J=7.5 \mathrm{~Hz}, 3 \mathrm{H})$; IR (KBr) $v$ : $1606.6 \mathrm{~cm}^{-1}$; HRMS calcd for $\mathrm{C}_{18} \mathrm{H}_{20} \mathrm{NS} 282.1311$, found 282.1312 .

7l: 白色固体, 产率 $83 \%$. m.p. $37 \sim 39{ }^{\circ} \mathrm{C}$; ${ }^{1} \mathrm{H}$ NMR $\left(500 \mathrm{MHz}, \mathrm{CDCl}_{3}\right) \delta: 7.06 \sim 8.05(\mathrm{~m}, 8 \mathrm{H}), 3.81 \sim 3.88(\mathrm{~m}$, $1 \mathrm{H}), 3.04(\mathrm{dd}, J=5.0,13.0 \mathrm{~Hz}, 1 \mathrm{H}), 2.50(\mathrm{dd}, J=12.0$, $13.0 \mathrm{~Hz}, 1 \mathrm{H}), 1.49 \sim 1.70(\mathrm{~m}, 3 \mathrm{H}), 1.36 \sim 1.45(\mathrm{~m}, 1 \mathrm{H})$, $0.93(\mathrm{t}, J=7.5 \mathrm{~Hz}, 3 \mathrm{H})$; IR (KBr) $v: 1608.5 \mathrm{~cm}^{-1}$; HRMS calcd for $\mathrm{C}_{18} \mathrm{H}_{19} \mathrm{FNS} 300.1217$, found 300.1217.

7m: 白色固体, 产率 86\%. m.p. $71 \sim 72{ }^{\circ} \mathrm{C} ;{ }^{1} \mathrm{H}$ NMR $\left(\mathrm{CDCl}_{3}, 500 \mathrm{MHz}\right) \delta: 7.07 \sim 7.99(\mathrm{~m}, 8 \mathrm{H}), 3.81 \sim$ $3.88(\mathrm{~m}, 1 \mathrm{H}), 3.04(\mathrm{dd}, J=5.0,13.0 \mathrm{~Hz}, 1 \mathrm{H}), 2.51(\mathrm{dd}, J=$ 11.0, $13.0 \mathrm{~Hz}, 1 \mathrm{H}), 1.61 \sim 1.67(\mathrm{~m}, 2 \mathrm{H}), 1.50 \sim 1.57(\mathrm{~m}$, $1 \mathrm{H}), 1.38 \sim 1.45(\mathrm{~m}, 1 \mathrm{H}), 0.93(\mathrm{t}, J=7.5 \mathrm{~Hz}, 3 \mathrm{H}) ; \mathrm{IR}$ (KBr) $v: 1616.2 \mathrm{~cm}^{-1}$; MS (30 eV) $\mathrm{m} / z(\%): 316.01(\mathrm{M}+$ $\mathrm{H}^{+}$, 100) 317.99 (50). Anal. calcd for $\mathrm{C}_{18} \mathrm{H}_{18} \mathrm{NSCl}$ : C 68.45, H 5.74, N 4.43; found C 68.49, H 5.55, N 4.61.

7n: 白色固体, 产率 $85 \%$. m.p. $78 \sim 79{ }^{\circ} \mathrm{C} ;{ }^{1} \mathrm{H}$ NMR $\left(\mathrm{CDCl}_{3}, 500 \mathrm{MHz}\right) \delta: 7.07 \sim 7.91(\mathrm{~m}, 8 \mathrm{H}), 3.81 \sim 3.87(\mathrm{~m}$, $1 \mathrm{H}), 3.03(\mathrm{dd}, J=5.5,13.0 \mathrm{~Hz}, 1 \mathrm{H}), 2.50(\mathrm{dd}, J=12.0$, $13.0 \mathrm{~Hz}, 1 \mathrm{H}), 1.50 \sim 1.67(\mathrm{~m}, 3 \mathrm{H}), 1.36 \sim 1.46(\mathrm{~m}, 1 \mathrm{H})$, 0.93 (t, $J=7.5 \mathrm{~Hz}, 3 \mathrm{H})$; IR (KBr) $v: 1604.7 \mathrm{~cm}^{-1}$; MS (70 eV) $m / z(\%): 360.00\left(\mathrm{M}+\mathrm{H}^{+}, 100\right), 362.95$ (46). Anal. calcd for $\mathrm{C}_{18} \mathrm{H}_{18} \mathrm{NSBr}$ : C 60.00, H 5.04, N 3.89; found C 60.02, H 5.13, N 3.78 .

7o: 白色固体, 产率 $82 \%$. m.p. $55 \sim 57{ }^{\circ} \mathrm{C} ;{ }^{1} \mathrm{H}$ NMR $\left(\mathrm{CDCl}_{3}, 500 \mathrm{MHz}\right) \delta: 7.04 \sim 7.93(\mathrm{~m}, 8 \mathrm{H}), 3.81 \sim 3.88(\mathrm{~m}$, $1 \mathrm{H}), 3.07(\mathrm{dd}, J=5.0,12.5 \mathrm{~Hz}, 1 \mathrm{H}), 2.50(\mathrm{dd}, J=11.5$, $12.5 \mathrm{~Hz}, 1 \mathrm{H}), 2.42(\mathrm{~s}, 3 \mathrm{H}), 1.60 \sim 1.67(\mathrm{~m}, 2 \mathrm{H}), 1.50 \sim$ $1.57(\mathrm{~m}, 1 \mathrm{H}), 1.38 \sim 1.45(\mathrm{~m}, 1 \mathrm{H}), 0.93(\mathrm{t}, J=7.5 \mathrm{~Hz}, 3 \mathrm{H})$; IR (KBr) $v: 1602.7 \mathrm{~cm}^{-1}$; MS (70 eV) $\mathrm{m} / z(\%): 296.14$ $\left(\mathrm{M}+\mathrm{H}^{+}, 53\right), 296.66$ (100). Anal. calcd for $\mathrm{C}_{19} \mathrm{H}_{21} \mathrm{NS}$ : C 77.24, H 7.16, N 4.74; found C 77.25, H 7.28, N 4.82.

7p: 白色固体, 产率 $89 \%$. m.p. $61 \sim 63{ }^{\circ} \mathrm{C} ;{ }^{1} \mathrm{H}$ NMR $\left(\mathrm{CDCl}_{3}, 500 \mathrm{MHz}\right) \delta: 6.97 \sim 8.02(\mathrm{~m}, 8 \mathrm{H}), 3.88(\mathrm{~s}, 3 \mathrm{H})$, $3.81 \sim 3.87(\mathrm{~m}, 1 \mathrm{H}), 3.06(\mathrm{dd}, J=5.0,12.5 \mathrm{~Hz}, 1 \mathrm{H}), 2.50$ (dd, $J=11.5,12.5 \mathrm{~Hz}, 1 \mathrm{H}), 1.62 \sim 1.67(\mathrm{~m}, 2 \mathrm{H}), 1.50 \sim$ $1.57(\mathrm{~m}, 1 \mathrm{H}), 1.38 \sim 1.46(\mathrm{~m}, 1 \mathrm{H}), 0.93(\mathrm{t}, J=7.5 \mathrm{~Hz}, 3 \mathrm{H})$; IR (KBr) $v: 1610.5 \mathrm{~cm}^{-1}$; MS (70 eV) $\mathrm{m} / \mathrm{z}(\%): 312.08$ $\left(\mathrm{M}+\mathrm{H}^{+}, 100\right), 323.07$ (40). Anal. calcd for $\mathrm{C}_{19} \mathrm{H}_{21} \mathrm{NS}: \mathrm{C}$ 73.27, H 6.80, N 4.50; found C 73.29, H 6.89, N 4.49.

7q: 白色固体, 产率 90\%. m.p. $109 \sim 110{ }^{\circ} \mathrm{C} ;{ }^{1} \mathrm{H}$
NMR $\left(\mathrm{CDCl}_{3}, 500 \mathrm{MHz}\right) \delta: 7.11 \sim 8.84(\mathrm{~m}, 8 \mathrm{H}), 3.87 \sim$ $3.93(\mathrm{~m}, 1 \mathrm{H}), 3.09(\mathrm{dd}, J=5.5,13.0 \mathrm{~Hz}, 1 \mathrm{H}), 2.58(\mathrm{dd}, J=$ $11.5,13.0 \mathrm{~Hz}, 1 \mathrm{H}), 1.65 \sim 1.70(\mathrm{~m}, 2 \mathrm{H}), 1.53 \sim 1.61(\mathrm{~m}$, $1 \mathrm{H}), 1.40 \sim 1.48(\mathrm{~m}, 1 \mathrm{H}), 0.95(\mathrm{t}, J=7.5 \mathrm{~Hz}, 3 \mathrm{H}) ; \mathrm{IR}$ (KBr) $v: 1602.7 \mathrm{~cm}^{-1}$; MS (70 eV) $\mathrm{m} / z(\%): 327.03(\mathrm{M}+$ $\left.\mathrm{H}^{+}, 100\right), 328.04$ (20). Anal. calcd for $\mathrm{C}_{18} \mathrm{H}_{18} \mathrm{~N}_{2} \mathrm{O}_{2} \mathrm{~S}: \mathrm{C}$ 66.23, H 5.56, N 8.58; found C 66.20, H 5.67, N 8.59.

7r: 白色固体, 产率 84\%. m.p. 67 $69{ }^{\circ} \mathrm{C} ;{ }^{1} \mathrm{H}$ NMR $\left(\mathrm{CDCl}_{3}, 500 \mathrm{MHz}\right) \delta: 7.04 \sim 8.02(\mathrm{~m}, 8 \mathrm{H}), 3.83 \sim 3.89(\mathrm{~m}$, $1 \mathrm{H}), 3.10(\mathrm{dd}, J=5.0,13.0 \mathrm{~Hz}, 1 \mathrm{H}), 2.49(\mathrm{dd}, J=11.5$, $13.0 \mathrm{~Hz}, 1 \mathrm{H}), 1.61 \sim 1.66(\mathrm{~m}, 2 \mathrm{H}), 1.49 \sim 1.56(\mathrm{~m}, 1 \mathrm{H})$, $1.37 \sim 1.43(\mathrm{~m}, 1 \mathrm{H}), 0.93(\mathrm{t}, J=7.5 \mathrm{~Hz}, 3 \mathrm{H})$; IR $(\mathrm{KBr}) v$ : $1610.5 \mathrm{~cm}^{-1}$; MS (30 eV) m/z (\%): $315.82\left(\mathrm{M}+\mathrm{H}^{+}, 95\right)$ 317.81 (100). Anal. calcd for $\mathrm{C}_{18} \mathrm{H}_{18} \mathrm{NSCl}$ : C $68.45, \mathrm{H}$ 5.74, N 4.43; found C 68.46, H 5.55, N 4.52.

7s: 白色固体, 产率 82\%. m.p. $103 \sim 105{ }^{\circ} \mathrm{C} ;{ }^{1} \mathrm{H}$ NMR $\left(\mathrm{CDCl}_{3}, 500 \mathrm{MHz}\right) \delta: 7.04 \sim 8.04(\mathrm{~m}, 7 \mathrm{H}), 3.80 \sim$ $3.87(\mathrm{~m}, 1 \mathrm{H}), 3.06(\mathrm{dd}, J=5.5,12.5 \mathrm{~Hz}, 1 \mathrm{H}), 2.48(\mathrm{dd}, J=$ $12.0,12.5 \mathrm{~Hz}, 1 \mathrm{H}), 1.59 \sim 1.67(\mathrm{~m}, 2 \mathrm{H}), 1.49 \sim 1.56(\mathrm{~m}$, $1 \mathrm{H}), 1.38 \sim 1.44(\mathrm{~m}, 1 \mathrm{H}), 0.93(\mathrm{t}, J=7.5 \mathrm{~Hz}, 3 \mathrm{H}) ; \mathrm{IR}$ (KBr) $v: 1614.4 \mathrm{~cm}^{-1}$; HRMS calcd for $\mathrm{C}_{18} \mathrm{H}_{18} \mathrm{FCINS}$ 334.0827, found 334.0830 .

7t: 白色固体, 产率 $83 \%$. m.p. $100 \sim 102{ }^{\circ} \mathrm{C} ;{ }^{1} \mathrm{H}$ NMR $\left(\mathrm{CDCl}_{3}, 500 \mathrm{MHz}\right) \delta: 7.07 \sim 7.99(\mathrm{~m}, 7 \mathrm{H}), 3.81 \sim$ $3.87(\mathrm{~m}, 1 \mathrm{H}), 3.07(\mathrm{dd}, J=5.0,12.5 \mathrm{~Hz}, 1 \mathrm{H}), 2.49(\mathrm{dd}, J=$ $12.0,12.5 \mathrm{~Hz}, 1 \mathrm{H}), 1.59 \sim 1.67(\mathrm{~m}, 2 \mathrm{H}), 1.49 \sim 1.56(\mathrm{~m}$, 1H), $1.37 \sim 1.44(\mathrm{~m}, 1 \mathrm{H}), 0.93(\mathrm{t}, J=7.5 \mathrm{~Hz}, 3 \mathrm{H})$; IR (KBr) $v$ : $1612.5 \mathrm{~cm}^{-1}$; MS (30 eV) $\mathrm{m} / z(\%): 350.05(\mathrm{M}+$ $\left.\mathrm{H}^{+}, 100\right), 352.05$ (68). Anal. calcd for $\mathrm{C}_{18} \mathrm{H}_{17} \mathrm{NSCl}_{2}$ : C 61.72, H 4.89, N 4.00; found C 61.74, H 4.78, N 4.21.

7u: 白色固体, 产率 85\%. m.p. $75 \sim 78{ }^{\circ} \mathrm{C} ;{ }^{1} \mathrm{H}$ NMR $\left(\mathrm{CDCl}_{3}, 500 \mathrm{MHz}\right) \delta: 7.04 \sim 7.89(\mathrm{~m}, 7 \mathrm{H}), 3.80 \sim 3.86(\mathrm{~m}$, $1 \mathrm{H}), 3.03$ (dd, $J=5.0,12.5 \mathrm{~Hz}, 1 \mathrm{H}), 2.47$ (dd, $J=12.0$, $12.5 \mathrm{~Hz}, 1 \mathrm{H}), 1.59 \sim 1.67(\mathrm{~m}, 2 \mathrm{H}), 1.49 \sim 1.58(\mathrm{~m}, 1 \mathrm{H})$, $1.36 \sim 1.45(\mathrm{~m}, 1 \mathrm{H}), 0.93(\mathrm{t}, J=7.0 \mathrm{~Hz}, 3 \mathrm{H})$; IR (KBr) $v$ : $1610.5 \mathrm{~cm}^{-1}$; MS (30 eV) $\mathrm{m} / \mathrm{z}(\%): 393.92\left(\mathrm{M}+\mathrm{H}^{+}, 83\right)$, 395.91 (100). Anal. calcd for $\mathrm{C}_{18} \mathrm{H}_{17} \mathrm{NSBrCl}$ : C 54.77, $\mathrm{H}$ 4.34, N 3.55; found C 54.75, H 4.39, N 3.66.

7v: 白色固体, 产率 86\%. m.p. 77 78 ${ }^{\circ} \mathrm{C} ;{ }^{1} \mathrm{H}$ NMR $\left(\mathrm{CDCl}_{3}, 500 \mathrm{MHz}\right) \delta: 7.03 \sim 7.93(\mathrm{~m}, 7 \mathrm{H}), 3.81 \sim 3.87(\mathrm{~m}$, $1 \mathrm{H}), 3.09$ (dd, $J=5.5,12.5 \mathrm{~Hz}, 1 \mathrm{H}), 2.48(\mathrm{dd}, J=11.5$, $12.5 \mathrm{~Hz}, 1 \mathrm{H}), 2.43(\mathrm{~s}, 3 \mathrm{H}), 1.61 \sim 1.66(\mathrm{~m}, 2 \mathrm{H}), 1.49 \sim$ $1.56(\mathrm{~m}, 1 \mathrm{H}), 1.37 \sim 1.44(\mathrm{~m}, 1 \mathrm{H}), 0.93(\mathrm{t}, J=7.5 \mathrm{~Hz}, 3 \mathrm{H})$; IR (KBr) $v: 1608.5 \mathrm{~cm}^{-1}$; MS (30 eV) $\mathrm{m} / z(\%): 330.1$ $\left(\mathrm{M}+\mathrm{H}^{+}, 100\right)$. Anal. calcd for $\mathrm{C}_{19} \mathrm{H}_{20} \mathrm{NSCl}$ : C 69.18, H 
6.11, N 4.25; found C 69.22, H 6.23, N 4.29.

7w: 白色固体，产率 $82 \%$. m.p. $88 \sim 90{ }^{\circ} \mathrm{C} ;{ }^{1} \mathrm{H}$ NMR $\left(\mathrm{CDCl}_{3}, 500 \mathrm{MHz}\right) \delta: 6.97 \sim 8.01(\mathrm{~m}, 7 \mathrm{H}), 3.89(\mathrm{~s}$, $3 \mathrm{H}), 3.79 \sim 3.86(\mathrm{~m}, 1 \mathrm{H}), 3.08(\mathrm{dd}, J=5.5,13.0 \mathrm{~Hz}, 1 \mathrm{H})$, $2.47(\mathrm{dd}, J=11.5,13.0 \mathrm{~Hz}, 1 \mathrm{H}), 1.61 \sim 1.66(\mathrm{~m}, 2 \mathrm{H})$, $1.49 \sim 1.56(\mathrm{~m}, 1 \mathrm{H}), 1.37 \sim 1.45(\mathrm{~m}, 1 \mathrm{H}), 0.93(\mathrm{t}, J=7.5$ $\mathrm{Hz}, 3 \mathrm{H})$; IR (KBr) v: $1608.5 \mathrm{~cm}^{-1}$; MS (30 eV) $\mathrm{m} / z(\%)$ : $346.02\left(\mathrm{M}+\mathrm{H}^{+}, 100\right), 348.00$ (40). Anal. calcd for $\mathrm{C}_{19} \mathrm{H}_{20} \mathrm{NOSCl}$ : C 65.98, H 5.83, N 4.05; found C 65.96, H 5.94, N 4.14.

7x: 白色固体，产率 $89 \%$. m.p. $140 \sim 142{ }^{\circ} \mathrm{C} ;{ }^{1} \mathrm{H}$ NMR $\left(\mathrm{CDCl}_{3}, 500 \mathrm{MHz}\right) \delta: 7.09 \sim 8.82(\mathrm{~m}, 7 \mathrm{H}), 3.86 \sim$ $3.92(\mathrm{~m}, 1 \mathrm{H}), 3.11(\mathrm{dd}, J=5.0,13.0 \mathrm{~Hz}, 1 \mathrm{H}), 2.56(\mathrm{dd}, J=$ $11.5,13.0 \mathrm{~Hz}, 1 \mathrm{H}), 1.61 \sim 1.71(\mathrm{~m}, 2 \mathrm{H}), 1.52 \sim 1.58(\mathrm{~m}$, $1 \mathrm{H}), 1.39 \sim 1.47(\mathrm{~m}, 1 \mathrm{H}), 0.95(\mathrm{t}, J=7.5 \mathrm{~Hz}, 3 \mathrm{H})$; IR $(\mathrm{KBr}) v: 1608.5 \mathrm{~cm}^{-1}$; MS (30 eV) $\mathrm{m} / z(\%): 361.00(\mathrm{M}+$ $\left.\mathrm{H}^{+}, 100\right), 362.99$ (50). Anal. calcd for $\mathrm{C}_{18} \mathrm{H}_{17} \mathrm{~N}_{2} \mathrm{O}_{2} \mathrm{SCl}$ : C 59.91, H 4.75, N 7.76; found C 59.73, H 4.71, N 7.57.

\section{References}

[1] Nagao, T.; Sato, M.; Nakajima, H.; Kiyomoto, A. Chem. Pharm. Bull. 1973, 2l(1), 92.

[2] Maruenda, H.; Johnson, F. J. Med. Chem. 1995, 38, 2145.

[3] Disanto, R.; Costi, R. Farmaco 2005, 60, 385.
[4] Garg, N.; Chandra, T.; Archana, J. A. B.; Kumar, A. Eur. J. Med. Chem. 2010, 45, 1529 .

[5] Ichikawa, M.; Yokomizo, A.; Itoh, M.; Sugita, K.; Usui, H.; Shimizu, H.; Suzuki, M.; Terayama, K.; Kanda, A. Bioorg. Med. Chem. 2011, 19, 1930.

[6] Karale, S. N.; Pratap, U. R.; Mahalle, S. R.; Mane, R. A. J. Sulfur Chem. 2011, 32(4), 303.

[7] Dandia, A.; Singh, R.; Singh, D.; Laxkar, A.; Sivpuri, A. Phosphorus, Sulfur, Silicon Relat. Elem. 2010, 185, 2472.

[8] Chate, A. V.; Joshi, R. S.; Badadhe, P. V.; Dabhade, S. K.; Gill, C. H. Bull. Korean Chem. Soc. 2011, 32(11), 3887.

[9] Ghotekar, D. S; Joshi, R. S.; Mandhane, P. G.; Bhagat, S. S.; Gill, C. H. Indian J. Chem. 2010, 49B(9), 1267.

[10] Zhang, P.; Xuan, Y.; Liu, W.; Li, Y. Chin. J. Org. Chem. 2011, 31, 1240 (in Chinese)

(张萍, 宣烨, 刘薇, 李媛, 有机化学, 2011, 31, 1240.)

[11] Li, W.-H.; Zhang, L.-F.; Liu, W.; Du, X.-Q.; Li, Y. Chin. J. Org. Chem. 2011, 31, 1252 (in Chinese).

(李文红, 张玲芬, 刘薇, 杜星琼, 李媛, 有机化学, 2011，31, 1252.)

[12] Wu, H.-S. M.S. Thesis, Hebei Normal University, Shijianzhuang, 2008 (in Chinese). (吴惠姝，硕士论文，河北师范大学，石家庄, 2008.)

[13] Anand, R. C.; Selvapalam, N. Synth. Commun. 1994, 24, 2743.

[14] Gannett, P. M.; Nagel, D. L.; Reilly, P. J.; Lawson, T.; Sharpe, J.; Toth, B. J. Org. Chem. 1988, 53(5), 1064.

[15] Yang, S.-X.; Yang, X.-L.; Lu, Y.-Y.; Sun, Y.-F.; Sun, L.; Ling, Y. Chin. J. Org. Chem. 2010, 30, 1543 (in Chinese)

(杨绍祥, 杨新玲, 卢园园, 孙玉风, 孙亮, 凌云, 有机化学, 2010, 30, 1543.) 